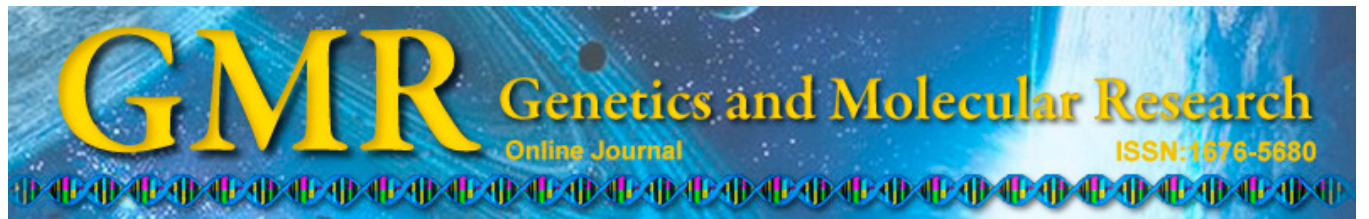

\title{
Evaluation of concentrations of botulinum toxin A for the treatment of hemifacial spasm: a randomized double-blind crossover trial
}

\author{
Y.J. Li, Y. Huang, Q. Ding, Z.H. Gu and X.L. Pan \\ Guangdong Province, China \\ Corresponding author: Y.J. Li \\ E-mail: youjialicn@163.com \\ Genet. Mol. Res. 14 (1): 1136-1144 (2015) \\ Received May 28, 2015 \\ Accepted August 14, 2014 \\ Published February 6, 2015 \\ DOI http://dx.doi.org/10.4238/2015.February.6.17
}

Department of Neurology, the First People's Hospital of Zhaoqing, Zhaoqing,

\begin{abstract}
The aim of our study was to evaluate the efficacy and safety of two concentrations of botulinum toxin A (BTX-A) for the treatment of hemifacial spasm. We randomly divided 20 patients with hemifacial spasm into high- and low-concentration groups; they were administered 50 and 25 U/mL BTX-A injection, respectively. Further, we compared the curative effects and the occurrence of adverse reactions in the two groups. Our results showed that both the concentrations of BTX-A were effective and no significant difference was observed in the onset time and therapeutic efficacy between the two groups; however, the duration of efficacy was longer in the high-concentration group than in the low-concentration group. Patients in both groups had no allergic reactions and systemic toxic reactions, but those in the highconcentration group had more serious adverse reactions and they lasted for longer durations. The adverse reactions in the two groups were not specifically treated, and they resolved in a relatively short time. In conclusion, local injection of BTX-A was effective in treating hemifacial spasm and each concentration of BTX-A had advantages and disadvantages, which indicated that the concentration of BTX-A
\end{abstract}


can be selected according to the clinical characteristics and willingness of the patients.

Key words: Botulinum toxin A; Dilution; Hemifacial spasm; Randomized controlled trial

\section{INTRODUCTION}

Hemifacial spasm is a type of chronic focal dystonia, and the pathogenesis of this disease remains to be clarified because it is caused by a variety of reasons (Campos-Benitez and Kaufmann, 2008; Han et al., 2009; Guan et al., 2011). Although hemifacial spasm is commonly observed in clinical practice, treatment for this disease remains a challenge for neurologists. Currently, no treatment is available for preventing or cure all hemifacial spasm patients. Hemifacial spasm is mainly characterized by recurrent involuntary twitching of muscles on one side of the face innervated by the facial nerve. During the early period, the disease is marked by intermittent convulsions of the orbicularis oculi muscle, which gradually spread to the other facial muscles the on same side. Twitching is aggravated when the patients are emotional and nervous and is reduced when the patients are calm and rested. Although hemifacial spasm is occasionally bilateral, it does not occur at the same time on each side. The clinical characteristics of the disease are that it is unilateral, involuntary, paroxysmal, painless, and irregular (Batla et al., 2012). Although the disease progresses slowly and is not life threatening, the recurrent cramps seriously affect the patient's social communication and quality of life, which results in anxiety, depression, and other affective disorders in many patients (Rudzińska et al., 2012). Many treatments are available for hemifacial spasm, and they include oral drug therapy, physical therapy, or surgery. Oral medications do not have optimum effects and are often accompanied with several side effects, physical therapy only decreases the onset of the disease to a certain extent for a short duration, and although surgical therapy has some curative effects, it may cause serious trauma and complications, has certain risks, and the disease may recur in some patients (Hyun et al., 2010; Ray et al., 2010).

Botulinum toxin is a neurotropic protein that is locally injected to relieve muscle spasm through a blockade of neuromuscular transmission. Different types of botulinum toxins have different times of onset of action. Currently, botulinum toxin A (BTX-A) has widespread clinical applications, and different preparations of BTX-A have shown no significant differences in treatment efficacy and side effects (Wu et al., 2011). In 1980, Dr. Scott of the USA reported the application of BTX-A for the first time after successful treatment of strabismus using a local injection of BTX-A (Scott, 1980). Subsequently, this therapy was used for dystonia (Barbosa et al., 2010; Gill and Kraft, 2010; Wu et al., 2011; Van Der Walt et al., 2012; Czyz et al., 2013). Since the 1990s, local injection of BTX-A has been widely used in the treatment of hemifacial spasm in China and abroad and has shown good results, which was considered to be a major development in the field of neurological treatment (Simpson et al., 2008; Barbosa et al., 2010; Wu et al., 2011; Czyz et al., 2013). However, the concentrations used to treat hemifacial spasms are different at different institutions, and thus, consistent results about the efficacy and incidence of side effects have not been reported thus far (Simpson et al., 2008; Barbosa et al., 2010; Czyz et al., 2013; Mazlout et al., 2013). Majority of the previous clinical studies were retrospective and nonrandomized, with no control group and a low level of evidence; therefore, definite conclusions cannot be obtained (Simpson et al., 2008; Nestor 
and Ablon, 2011; Mazlout et al., 2013). There are few randomized, controlled trials with appropriate designs and reliable quality, and prospective randomized trials need to be performed urgently to determine the clinical effect of BTX-A.

To observe the efficacy and safety of different concentrations of BTX-A for the treatment of hemifacial spasm, we designed a randomized double-blind crossover trial in patients with hemifacial spasm. We used non-electromyography (EMG)-guided freehand positioning injection method and different concentrations of a local injection of BTX-A for the treatment of hemifacial spasm. The patients were followed for 1 year to provide some references for clinical drug safety and choice of indications.

\section{MATERIAL AND METHODS}

\section{Subjects}

We enrolled patients with hemifacial spasms who visited our clinic between November 2007 and December 2010. This study was conducted in accordance with the Declaration of Helsinki and was approved by the Ethics Committee of the First People's Hospital of Zhaoqing. Written informed consent was obtained from all participants.

The patient inclusion criteria were as follows: 1) patients with a hemifacial spasm of strength $\geq 2$ [the spasm strength was assessed according to grade criteria of Cohen et al. (1986)]; 2) patients who had received oral medication or surgery but showed poor efficacy or were unable to tolerate these treatments or had a recurrence after the surgical treatment; 3) patients with a better compliance; and 4) patients who signed the informed consent. We enrolled male and female patients of all ages.

We excluded patients with a recent episode of fever and acute infectious diseases; allergic reactions; blood diseases; serious abnormal functioning of the heart, liver, kidney, or other organs; myasthenia gravis, Lambert-Eaton syndrome, motor neuron disease; epilepsy; peripheral facial paralysis with other diseases; and acute or other diseases that were difficult to control. Further, we excluded pregnant women and patient using aminoglycoside antibiotics or other medicines that might influence the neuromuscular transmission.

Trial termination criteria were serious or irreversible adverse reactions caused by the injection of BTX-A during the treatment.

\section{Treatment methods}

We performed a randomized double-blind crossover trial. According to the random number table method, the eligible patients were divided into a high-concentration group (50 $\mathrm{U} / \mathrm{mL}$ ) or a low-concentration group $(25 \mathrm{U} / \mathrm{mL})$, subsequently, the patients were crossed over to the opposite group and injected with BTX-A. We used the double-blind method to evaluate the efficacy in patients. BTX-A injection was provided by the Lanzhou Institute of Biological Products of China's Ministry of Health and was stored between $-5^{\circ} \mathrm{C}$ and $-20^{\circ} \mathrm{C}$ in a refrigerator. BTX-A was diluted with $9 \mathrm{~g} / \mathrm{L}$ sodium chloride solution to the concentration required (50 $\mathrm{U} / \mathrm{mL}$ or $25 \mathrm{U} / \mathrm{mL}$ ) before use. The preparation was injected within 1 to $4 \mathrm{~h}$ after preparation. Before treatment, the condition of the patients was diagnosed by a physician, and other antispasticity drugs were not generally used during the treatment. Patients were sitting or lying down, and 2.5 to 5 U BTX-A was subcutaneously or intramuscularly injected at each point 
using 1-mL skin test syringes and 4.5-mm needles; we used a non-EMG-guided manual positioning injection technique and a multi-point injection method, which prevented injecting into the blood vessel; each injection site was $2-4 \mathrm{~cm}$ apart. In a previous study, we showed that the longest symptom-free duration after local intramuscular injection of BTX-A was 11 months (Ray et al., 2010). We designed the study so that the washout period for the drug was 12 months, and for patients with recurrence, the interval between the previous and the subsequent treatment was 12 months.

\section{Observation indices}

In accordance with the unified form, we measured the muscle spasm strength before and after the treatment, onset time, time to peak effect, duration of the muscle spasm relief, and adverse reactions. After the treatment, patients were followed up at 1 week and at 1, 2, 3, $4,5,6$, and 12 months.

\section{Assessment of treatment efficacy and safety}

Spasm strength was classified according to Cohen grade criteria. A decrease in the Cohen score of $\geq 1$ grade was defined as effective treatment; onset time was defined as the number of days required for the Cohen score to decrease by $>1$ grade; and recurrence was defined as an increase in the spasm strength by $\geq 1$ grade. For the assessment of safety, we observed the incidences of adverse reactions and their duration after treatment with BTX-A.

\section{Statistical analysis}

We used SPSS13.0 for statistical analysis; the statistical data are reported as means $\pm \mathrm{SD}$. Analysis of variance of the two-period, crossover study data was used to analyze the therapeutic effect and the onset time in the two groups. The incidences of adverse reactions were compared using the chi-square test. The Wilcoxon test was used to compare the duration of adverse reactions. Two-sided hypothesis test was set at $\alpha=0.05$.

\section{RESULTS}

\section{Baseline data}

None of the 20 patients ( 7 men and 13 women) included in this analysis were lost to follow-up (Cohen scores of some patients were obtained through a telephonic follow-up). The average age of the patients was $52.9 \pm 11.4$ years, the course of the disease was $4.35 \pm 3.12$ years, Cohen grade was $2.8 \pm 0.5$, and the dosage of BTX-A was $47.25 \pm 5.5 \mathrm{U}$. No difference was observed in the total BTX-A levels between the two groups $(\mathrm{P}>0.05)$. There was no statistically significant concentration treatment sequence between two groups $(\mathrm{P}>0.05)$.

\section{Comparison of efficacy between the two groups}

Both the groups showed good efficacy in 1-5 days after treatment with BTX-A injection, and no significant difference was observed in the onset time between the two groups 
(Table 1). Peak effect was observed within 1 week. Within 3 months, both groups showed a similar decrease in the Cohen score. This decrease lasted for 3-5 months. The effect lasted for a longer duration in the high-concentration group than in the low-concentration group (Table 2). After 6 months, all patients had recurrence, but 2 patients showed a lower Cohen score than that before treatment.

Table 1. Comparison between the onset times in the two groups.

\begin{tabular}{lcccc}
\hline Item & High-concentration group & Low-concentration group & $F$ & P \\
\hline Onset time (day) & 2.40 & 2.25 & 0.717 & 0.408 \\
\hline
\end{tabular}

Table 2. Comparison between the decrease in the Cohen scores in the two groups.

\begin{tabular}{|c|c|c|c|c|}
\hline Time after treatment & High-concentration group & Low-concentration group & $F$ & $P$ \\
\hline 1 week to 3 months & 2.25 & 2.20 & 0.36 & 0.556 \\
\hline Month 4 & 2.20 & 1.85 & 7.23 & 0.015 \\
\hline Month 5 & 0.60 & 0.25 & 5.444 & 0.031 \\
\hline Months 6-12 & 0.05 & 0.05 & 0 & 1.000 \\
\hline
\end{tabular}

\section{Comparison of the incidences of adverse reactions between the two groups}

Allergic reactions and systemic poisoning were not observed in the two groups. Fifteen of the 20 patients in the high-concentration group and 4 of the 20 patients in the low-concentration group had adverse reactions, which indicated that the incidences of adverse reactions were significantly higher in the high-concentration group than in the low-concentration group (75 vs 20\%, respectively; Table 3). Patients in both groups did not require additional treatment, and the adverse reactions completely disappeared after 3-8 weeks.

Table 3. Comparison between the incidences of adverse reactions in the two groups (case).

\begin{tabular}{lccccc}
\hline Group & Deviated mouth & Ptosis & Orbicular weakness & Epiphora & Total \\
\hline High-concentration group & 11 & 2 & 1 & 1 & 15 \\
Low-concentration group & 4 & 0 & 0 & 0 & 4 \\
P & 0.022 & & & $<0.0005$ \\
\hline
\end{tabular}

\section{Comparison of the duration of adverse reactions between the two groups}

Adverse reactions occurred in 1-5 after injection, and the onset time was similar between both the groups. The adverse reactions in the low-concentration group disappeared after 3-4 weeks, whereas those in some patients in the high-concentration group disappeared after 8 weeks; however, the duration of adverse reactions was significantly different between the groups (Table 4).

Table 4. Comparison between the duration of adverse reactions in the two groups (week).

\begin{tabular}{|c|c|c|c|c|c|}
\hline Group & Deviated mouth & Ptosis & Orbicular weakness & Epiphora & Total \\
\hline High-concentration group & $5.73 \pm 1.68$ & 6.0 & 6.0 & 4.0 & $5.67 \pm 1.49$ \\
\hline Low-concentration group & $3.50 \pm 0.58$ & 0 & 0 & 0 & $3.50 \pm 0.58$ \\
\hline $\mathrm{P}$ & 0.024 & & & & 0.014 \\
\hline
\end{tabular}




\section{DISCUSSION}

BTX-A is a bacterial exotoxin produced by the anaerobic bacteria Clostridium botulinum and is one of the most potent bacterial toxins to date. Local injection of BTX-A acts by blocking the presynaptic release of acetylcholine and thus relieves muscle spasm. The denervation effect of BTX-A is not permanent, and after the reconstruction of motor end-plate function, nerve conduction and muscle contraction force is gradually restored. Because the adverse effect is temporary, repeated injections can be administered (Harrison et al., 2011; Butera et al., 2013). Since the 1990s, BTX-A has been used for the treatment of hemifacial spasm. Local intramuscular injection of BTX-A has improved efficacy, and the improvement of clinical symptoms lasts for 3-8 months with few side effects, and thus, studies on BTX-A have increased (Rudzińska et al., 2010; Butera et al., 2013).

However, the specific concentrations of BTX-A used clinically in China (50 or $25 \mathrm{U} /$ $\mathrm{mL}$ ) are different from those used abroad for the treatment of hemifacial spasm; some studies have reported the use of $12.5 \mathrm{U} / \mathrm{mL}$, and large differences have also been observed in efficacy and adverse drug reactions. Most studies suggest that the effect of different concentrations of BTX-A injection is not different and that the high concentrations may be associated with a higher incidences of adverse reactions. Some studies indicate that high concentrations of BTX-A injection are more effective than the low concentrations, which can shorten the time interval between repeat injections and is more likely to produce anti-BTX-A antibodies and drug resistance. A majority of the clinical studies performed thus far are retrospective, and few prospective randomized controlled trials with a high level of evidence are available (Simpson et al., 2008; Butera et al., 2013; Mazlout et al., 2013).

The site of action of the BTX-A is the neuromuscular junction of the spasmodic muscle, the degree at which BTX-A binds to the presynaptic receptor of the neuromuscular junction determines the degree of improvement of hemifacial spasm. Therefore, theoretically, a higher dose of the local injection of BTX-A can cause a more obvious improvement in the symptoms of hemifacial spasm and thus have an improved clinical effect. However, most of the facial muscles are small, and when the local dose of BTX-A reaches a certain dose, it may penetrate to the adjacent muscles and cause an adverse reaction. BTX-A must be diluted before injection; however, the lower the concentration the poorer is the effect, and at high concentrations the drug easily spreads to the surrounding muscles and causes serious adverse effects (Harrison et al., 2011). However, low concentrations of BTX-A may not sufficiently relieve the spasm symptoms. The premise of an ideal treatment with BTX-A is ensuring efficacy and decreasing the side effects. Some studies suggest that injecting BTX-A within $4 \mathrm{~h}$ after dissolution is as effective as injecting it after 2 weeks of cryopreservation (Sloop et al., 1997). However, to ensure the curative effect and decrease the toxic side effects, we injected BTX-A within $1 \mathrm{~h}$ of dissolution. Previous studies indicate that intramuscular injection under EMG guidance or electrical stimulation is better, but it requires experience and positioning the patients is difficult (Ploumis et al., 2014). Because EMG guidance or electrical stimulation is relatively complex and the facial muscles are superficial, we used the non-EMG-guided manual positioning method for injection in this study.

Results from our randomized double-blind controlled trial showed that the application of two different concentrations of BTX-A in the treatment of hemifacial spasm had similar curative effects and rapid onset time, but the higher concentration produced effects that lasted for a longer duration. However, patients in both the high- and low-concentration groups showed 
recurrence after 6 months of treatment, which indicated that BTX-A is effective in the treatment of hemifacial spasm. The interval between repeated, injections was longer for the higher concentration and the antibodies were not produced easily, which decreased the number of needle punctures and repeated injections, the overall injection pain, and the economic burden.

BTX-A is not effective for the treatment of dystonia in some patients because of resistance; resistance occurs in 5-10\% patients and develops mostly because the body produces antibodies against BTX-A, and the reversal of resistance can be detected by extensor digitorum brevis experiment (Kessler and Benecke, 1997; Kenis et al., 2011; Coleman et al., 2012). In our study, none of the patients in both groups showed resistance to BTX-A, and the efficacy rate was $100 \%$, which was not consistent that reported in previous studies; this finding may be related to the long time between subsequent injections or the method of injection. In order to reduce the possible of BTX-A resistance phenomenon, it is crucial to follow strict operation regulations, administer appropriate dosages, and repeated injection schedule. The interval between two treatments should be at least 3 to 6 months; the injection should not be repeated within a short time because of poor efficacy.

Injecting BTX-A into facial muscles can cause irreversible local facial atrophy, and animal studies have shown that BTX-A can cause segmental irreversible atrophy in the extraocular muscle fibers at the injection site. In this study, 2 patients, one each from the high- and low-concentration group, showed recurrence with a Cohen score that was lower by 1 grade than that before the treatment. That pathogenesis remains to be clarified and may be related to denervation atrophy caused by BTX-A and may differ between individuals. After intramuscular injection of BTX-A, a part of the muscle may lose normal nerve nutrition, which may produce irreversible segmental atrophy in the muscle fibers. This can cause segmental hyperplasia, and then the entire strength of the local muscle cannot be restored (Harrison et al., 2011).

Our study showed that patients in both groups had no allergic reactions and systemic toxic reactions. The most common local adverse drug reactions were deviated mouth, ptosis, orbicularis weakness, and epiphora, which gradually appeared when the symptoms improved. High concentration of BTX-A showed more serious side effects and longer duration of side effects. However, the adverse reactions in both groups did not require special treatment, and they completely disappeared in a short period of time, which indicated that BTX-A may be safe for the treatment of hemifacial spasms. The side effects are mild, transient, and easy to accept for the patients if they have been explained about those effects before the treatment.

In conclusion, our study showed that local injection of BTX-A has therapeutic, fast acting, and long-lasting effects for the treatment of hemifacial spasm. The adverse effects associated with BTX-A treatment are mild and transient, and repeated injections can be administered. Both the concentrations have advantages and disadvantages. The high concentration is associated with a longer interval time between repeated injections, lower incidence of pain, and reduced economic burden, but has relatively obvious adverse effects for a longer duration. Clinical treatment can be selected according to the patient's condition and willingness. However, because of the small sample size and short-term follow-up, the differences in longterm efficacy and adverse reactions between the two concentrations of BTX-A in treatment of hemifacial spasm could not be clarified. Thus, further multi-center studies with a large sample size should be performed to clarify these differences. 


\section{ACKNOWLEDGMENTS}

Research jointly supported by the Guangdong Province Science and Technology Plan Project (project \#2010B031600031), the Guangdong Province Medical Research Foundation (project \#A2010651), and the Zhaoqing City Science and Technology Innovation Project (project \#2007E111).

\section{REFERENCES}

Barbosa ER, Takada LT, Gonçalves LR, Costa RM, et al. (2010). Botulinum toxin type A in the treatment of hemifacial spasm: an 11-year experience. Arq. Neuropsiquiatr. 68: 502-505.

Batla A, Goyal C, Shukla G, Goyal V, et al. (2012). Hemifacial spasm: clinical characteristics of 321 Indian patients. J. Neurol. 259: 1561-1565.

Butera C, Guerriero R, Amadio S, Ungaro D, et al. (2013). Functional end-plate recovery in long-term botulinum toxin therapy of hemifacial spasm a nerve conduction study. Neurol. Sci. 34: 209-215.

Campos-Benitez M and Kaufmann AM (2008). Neurovascular compression findings in hemifacial spasm. J. Neurosurg. 109: 416-420.

Cohen DA, Savino PJ, Stern MB and Hurtig HI (1986). Botulinum injection therapy for blepharospasm: a review and report of 75 patients. Clin. Neuropharmacol. 9: 415-429.

Coleman C, Hubble J, Schwab J, Beffy JL, et al. (2012). Immunoresistance in cervical dystonia patients after treatment with abobotulinumtoxinA. Int. J. Neurosci. 122: 358-362.

Czyz CN, Burns JA, Petrie TP, Watkins JR, et al. (2013). Long-term botulinum toxin treatment of benign essential blepharospasm, hemifacial spasm, and Meige syndrome. Am. J. Ophthalmol. 156: 173-177.

Gill HS and Kraft SP (2010). Long-term efficacy of botulinum a toxin for blepharospasm and hemifacial spasm. Can. J. Neurol. Sci. 7: 631-636.

Guan HX, Zhu J and Zhong J (2011). Correlation between idiopathic hemifacial spasm and the MRI characteristics of the vertebral artery. J. Clin. Neurosci. 18: 528-530.

Han IB, Chang JH, Chang JW, Huh R, et al. (2009). Unusual causes and presentations of hemifacial spasm. Neurosurgery 65: $130-137$.

Harrison AR, Berbos Z, Zaldivar RA, Anderson BC, et al. (2011). Modulating neuromuscular junction density changes in botulinum toxin-treated orbicularis oculi muscle. Invest. Ophthalmol. Vis. Sci. 52: 982-986.

Hyun SJ, Kong DS and Park K (2010). Microvascular decompression for treating hemifacial spasm: lessons learned from a prospective study of 1,174 operations. Neurosurg. Rev. 33: 325-334.

Kenis VM, Baindurashvili AG, Dovbeshko AG, Korol'kova TN, et al. (2011). Botulinum toxine treatment and the formation of antibodies to botulotoxin in patients with cerebral palsy. Zh. Nevrol. Psikhiatr. Im. S. S. Korsakova 111: 48-51.

Kessler KR and Benecke R (1997). The EBD test-a clinical test for the detection of antibodies to botulinum toxin type A. Mov. Disord. 12: 95-99.

Mazlout H, Kamoun Gargouri H, Triki W, Kéfi S, et al. (2013). Safety and efficacy of botulinum toxin in hemifacial spasm. J. Fr. Ophtalmol. 36: 242-246.

Nestor MS and Ablon GR (2011). Duration of action of abobotulinumtoxinA and onabotulinumtoxinA: a randomized, double-blind study using a contralateral frontalis model. J. Clin. Aesthet. Dermatol. 4: 43-49.

Ploumis A, Varvarousis D, Konitsiotis S and Beris A (2014). Effectiveness of botulinum toxin injection with and without needle electromyographic guidance for the treatment of spasticity in hemiplegic patients: a randomized controlled trial. Disabil. Rehabil. 36: 313-318.

Ray DK, Bahgat D, McCartney S and Burchiel KJ (2010). Surgical outcome and improvement in quality of life after microvascular decompression for hemifacial spasms: a case series assessment using a validated disease-specific scale. Stereotact. Funct. Neurosurg. 88: 383-389.

Rudzińska M, Wójcik M and Szczudlik A (2010). Hemifacial spasm nonmotor and motor-related symptoms and their response to botulinum toxin therapy. J. Neural. Transm. 117: 765-772.

Rudzińska M, Wójcik M, Malec M, Grabska N, et al. (2012). Factors affecting the quality of life in hemifacial spasm patients. Neurol. Neurochir. Pol. 46: 121-129.

Scott AB (1980). Botulinum toxin injection into extraocular muscles as an alternative to strabismus surgery. Ophthalmology 87: 1044-1049. 
Simpson DM, Blitzer A, Brashear A, Comella C, et al. (2008). Assessment: botulinum neurotoxin for the treatment of movement disorders (an evidence-based review): report of the Therapeutics and Technology Assessment Subcommittee of the American Academy of Neurology. Neurology 70: 1699-1706.

Sloop RR, Cole BA and Escutin RO (1997). Reconstituted botulinum toxin type A does not lose potency in humans if it is refrozen or refrigerated for 2 weeks before use. Neurology 48: 249-253.

Van Der Walt A, Sung S, Spelman T, Marriott M, et al. (2012). A double-blind, randomized, controlled study of botulinum toxin type A in MS-related tremor. Neurology 79: 92-99.

Wu CJ, Shen JH, Chen Y and Lian YJ (2011). Comparison of two different formulations of botulinum toxin A for the treatment of blepharospasm and hemifacial spasm. Turk. Neurosurg. 21: 625-629. 